\title{
Meta
}

Journal des traducteurs

Translators' Journal

\section{Mettre la main sur le figement lexical : la démarche du traducteur}

\section{Christine Durieux}

Volume 53, numéro 2, juin 2008

La traduction des séquences figées

The Translation of Frozen Sequences

URI : https://id.erudit.org/iderudit/018522ar

DOI : https://doi.org/10.7202/018522ar

Aller au sommaire du numéro

Éditeur(s)

Les Presses de l'Université de Montréal

ISSN

0026-0452 (imprimé)

1492-1421 (numérique)

Découvrir la revue

Citer cet article

Durieux, C. (2008). Mettre la main sur le figement lexical : la démarche du traducteur. Meta, 53(2), 324-332. https://doi.org/10.7202/018522ar

\section{Résumé de l'article}

Le figement lexical oppose une résistance particulière à la traduction du fait de son ancrage culturel profond et de son caractère métaphorique. En fait, le figement lexical présent dans le texte de départ pose un double problème au traducteur : identification et signification. D'une part, le traducteur doit reconnaître la séquence figée dans la langue étrangère pour en cerner le périmètre afin de la traiter globalement comme une seule unité signifiante et, d'autre part, il doit en construire la signification en prenant appui sur les indices présents dans le contexte et en les exploitant dans un processus inférentiel. Cette étude montre la dimension polysémique des séquences figées en développant l'exemple d'une séquence verbale usuelle : mettre la main sur. 


\title{
Mettre la main sur le figement lexical: la démarche du traducteur
}

\author{
CHRISTINE DURIEUX \\ Université de Caen, Caen, France \\ christine.durieux@unicaen.fr
}

\begin{abstract}
RÉSUMÉ
Le figement lexical oppose une résistance particulière à la traduction du fait de son ancrage culturel profond et de son caractère métaphorique. En fait, le figement lexical présent dans le texte de départ pose un double problème au traducteur: identification et signification. D'une part, le traducteur doit reconnaître la séquence figée dans la langue étrangère pour en cerner le périmètre afin de la traiter globalement comme une seule unité signifiante et, d'autre part, il doit en construire la signification en prenant appui sur les indices présents dans le contexte et en les exploitant dans un processus inférentiel. Cette étude montre la dimension polysémique des séquences figées en développant l'exemple d'une séquence verbale usuelle: mettre la main sur.
\end{abstract}

\begin{abstract}
Set phrases put up resistance to translation because they are deeply rooted in culture and contain a metaphoric value. Set phrases existing in a source text are a dual problem for the translator: the translator must (1) recognize the set phrase in a text written in a foreign language and belonging to a foreign culture, and determine its limits in order to process it globally as one signifying unit, and (2) build its meaning by interpreting hints present in the context and conducting an inference process. This study shows the polysemous dimension of set phrases using the example of a usual phrasal verb in French: mettre la main sur.
\end{abstract}

MOTS-CLÉS/KEYWORDS

figement, métaphore, processus inférentiel, séquence polylexicale

«Il faut, absolument parlant, emprunter des métaphores modérées à des allusions convenablement énigmatiques; car les métaphores sont des allusions, et c'est à quoi l'on reconnaît que la métaphore a été bien choisie.»

Aristote, Rhétorique, Livre III [1405b].

\section{Introduction}

Si le figement lexical présente une difficulté majeure dans la description des langues (Mejri 1997), force est de constater qu'il oppose une résistance à la traduction. Ce n'est pas l'impossibilité de transcoder d'une langue à l'autre les unités composant l'expression figée qui constitue l'obstacle. En tout état de cause, la traduction humaine ne procède jamais par substitution d'un code linguistique à un autre, mais implique le déroulement de tout un processus cognitif qui consiste pour le traducteur à comprendre un texte/discours pour le faire comprendre à des destinataires qui n'ont pas accès à l'original.

Conformément aux règles de déontologie, le traducteur professionnel travaille vers sa langue maternelle. Il en résulte que la première phase de l'opération tradui- 
sante - dont évidemment dépend toute la suite du processus et in fine la qualité de la traduction - consiste à comprendre le texte en langue étrangère. Or, c'est là que le figement lexical présent dans le texte de départ pose un double problème au traducteur: identification et signification. En effet, d'une part, le traducteur doit reconnaître la séquence figée dans la langue étrangère pour en cerner le périmètre afin de la traiter globalement comme une seule unité signifiante et, d'autre part, il doit en construire la signification en prenant appui sur des indices présents dans le contexte et en les exploitant dans un processus inférentiel. Ensuite, une fois que l'appréhension du sens de l'énoncé contenant l'expression figée est réalisée, lors de la seconde phase de l'opération traduisante, le traitement de la séquence figée se fait dans les mêmes conditions que la traduction de formes unilexicales: réexpression dans la langue d'arrivée du sens construit, avec l'effet recherché de nature à produire sur le lecteur l'impact conforme à la mission du texte (Durieux 1991). En conséquence, le présent développement porte principalement sur la démarche de compréhension permettant au traducteur de réaliser une traduction satisfaisante. À cet effet, l'exemple choisi est une séquence verbale usuelle: mettre la main sur (quelqu'un ou quelque chose).

\section{Corpus}

Pour la présente étude, un corpus de douze extraits de textes publiés comportant la séquence verbale mettre la main sur (quelqu'un ou quelque chose) a été constitué. Dans chacun des extraits, l'emploi de cette séquence polylexicale renvoie à la signification d'un verbe simple différent. Les extraits sont numérotés de sorte que chaque emploi puisse être traité commodément par la suite.

[1] Le but de l'épreuve est de simuler les tâches essentielles du pompier lorsqu'il doit monter des escaliers muni de tous ses vêtements de protection et transportant un boyau enroulé et le matériel de lutte contre l'incendie.

Il est permis au candidat de mettre la main sur le mur pour maintenir son équilibre, mais si elle y demeure longtemps ou si le mur devient un accotoir, un mot d'avertissement est donné au candidat.

Test de capacité physique - Recrutement des pompiers.

[2] Je pouvais bien prendre Albertine sur mes genoux, mettre la main sur ses cheveux, mais, comme si j'eusse manié une pierre qui enferme la saline des océans immémoriaux ou le rayon d'une étoile, je sentais que je touchais seulement l'enveloppe close d'un être qui, par l'intérieur, accédait à l'infini.

M. Proust, À la recherche du temps perdu.

[3] Les troubles de la mémoire commencent par se manifester dans des situations courantes de la vie quotidienne: un nom qui échappe, les lunettes que l'on cherche désespérément, les clés sur lesquelles on ne parvient plus à mettre la main [...]

La Recherche, novembre 1997, Alzheimer: la maladie du siècle.

[4] Au fond, sa pensée dut être que sa sœur n'attirait le vieux que dans le calcul de mettre la main sur le magot soupçonné.

É. Zola, La Terre, III.

[5] Nouveaux rebondissements dans le feuilleton Marionnaud. Mettant à profit la forte chute du cours de la Bourse, consécutive à la publication des résultats semestriels en perte de 79 millions d'euros, Albert Frère s'est invité au capital de la chaîne de parfumeries. Finalement, c'est un groupe chinois de Hong Kong, AS Watson (Hutchinson), qui a réussi à mettre la main sur le premier distributeur de parfums en Europe. Le Revenu, 7-13 janvier 2005. 
[6] Au niveau mondial, les grands groupes financiers, tels Suez et Vivendi, veulent mettre la main sur l'eau, la santé, l'agriculture, l'énergie, y voyant des sources potentielles de profits.

Le Revenu, 5-11 novembre 2004.

[7] Démétrios Poliorcète, fils d'Antigone, fut très tôt associé aux entreprises de son père et aux efforts de celui-ci pour conserver une grande partie de l'empire d'Alexandre. [...]. Après la mort d'Antigone à Ipsos, privé désormais de l'empire asiatique de son père, il se maintint en Grèce et dans les îles. Il réussit à mettre la main sur la Macédoine en 294.

C. Mossé, ALEXANDRE La destinée d'un mythe.

[8] Il n'est pas certain que les pays asiatiques conserveront à long terme leur place prééminente dans la recherche sur les cellules souches. Une question majeure se pose: les universités locales seront-elles en mesure de produire un nombre suffisant de chercheurs de pointe, le recrutement de chercheurs étrangers ne pouvant se poursuivre indéfiniment?

[...] Les électeurs californiens se sont prononcés en faveur de la proposition 71 aux termes de laquelle les scientifiques menant ce type de recherche en Californie bénéficieront d'une aide de l'État de 300 millions de dollars par an. Les pays d'Asie auront alors plus de mal à mettre la main sur l'élite des scientifiques.

Le Point, 13 janvier 2005.

[9] L’an dernier, les douanes françaises ont mis la main sur 458 kilos de caviar de contrebande en provenance de Russie. La plupart des trafiquants qui transportent la marchandise dans leurs valises se font pincer à la descente de l'avion à Roissy ou à Nice, qui assurent des liaisons directes avec Moscou.

Le Point, 16 décembre 2004.

[10] Déclaré ennemi public numéro un des États-Unis, Abou Moussab Al-Zarqaoui est considéré comme le chef de file le plus actif de la nébuleuse Al-Qaeda. Washington offre 25 millions de dollars à qui permettra de mettre la main sur le terroriste jordanien.

Le Point, 11 novembre 2004.

[11] Ce lundi, il va être bien difficile de mettre la main sur un pédiatre. Avec l'épidémie de bronchiolite qui sévit actuellement, les Urgences des hôpitaux vont être encore saturées.

Journal télévisé, 13 heures, 3 janvier 2005 (à propos de la journée de grève des pédiatres).

[12] À la fin du siècle dernier, vivait un homme de science, une éminence experte dans toutes les branches de la philosophie naturelle. [...]. Son entourage croyait à la possibilité de faire monter d'un jalon à l'autre une intelligence aussi puissante jusqu'à ce que le philosophe puisse mettre la main sur le secret de la force créatrice et fasse peut-être des mondes nouveaux.

N. Hawthorne, La Marque de naissance.

\section{Approche descriptive}

Les douze emplois proposés ci-dessus témoignent de la polysémie des séquences polylexicales figées. Il est certes difficile de mesurer le caractère polysémique de ces séquences et de les comparer à celui des éléments unilexicaux, mais la réflexion sur la construction du sens conduit à poser l'hypothèse d'une grande similitude de charge polysémique dans les unités polylexicales et unilexicales. 
Le tableau ci-dessous liste les douze emplois de la séquence verbale polylexicale choisie - mettre la main sur - et mentionne l'actualisation (+) ou non (-) des traits inhérents et des traits afférents. On remarque que les traits inhérents sont consignés dans la langue, comme l'indiquent les définitions des dictionnaires:

Main : organe de préhension et de tact qui termine les deux bras de l'homme en se divisant en cinq doigts, et qui sert à tenir, prendre, toucher, donner, recevoir, ...

Grand Larousse Encyclopédique, Main.

Main : partie du corps humain, organe de la préhension et du toucher, placé à l'extrémité du bras et muni de cinq doigts dont l'un est opposable aux autres.

Le Grand Robert de la langue française, Main.

Ici, pour les traits inhérents, on retiendra les sèmes: prolongement du bras (nature), toucher et préhension (fonctions). Des traits afférents lexicalisés viennent s'y ajouter sous la pression de l'usage et d'un consensus social. Ici, on retiendra les sèmes: possession, confiscation, découverte. Outre les traits inhérents et afférents, on aurait pu ajouter un autre trait discriminant: l'objet. En effet, en [8], [10] et [11], il s'agit de mettre la main sur des personnes, alors que dans toutes les autres occurrences, l'objet de l'action est une chose. Enfin, le contexte contribue à actualiser tel ou tel trait: «On dira, en effet, qu'un trait est actualisé quand la compétence interprétative reconnaît sa pertinence; or sa pertinence peut dépendre de sa récurrence en contexte» (Rastier 1996: 81).

Par exemple, en [5], le contexte financier/boursier est explicite et annonce l'OPA lancée par le groupe Hutchinson sur la société Marionnaud. Les traits inhérents sont totalement virtualisés et seuls les traits afférents, possession et confiscation, se trouvent actualisés.

\begin{tabular}{|c|c|c|c|c|c|c|c|}
\hline & \multicolumn{2}{|c|}{ Traits inhérents } & & \multicolumn{2}{c|}{ Traits afférents } & & Verbe simple \\
\hline & prolong bras & toucher & préhension & possession & confiscation & découverte & \\
\hline & & & & & & & \\
\hline 1 & + & + & - & - & - & - & s'appuyer \\
\hline 2 & + & + & - & - & - & - & caresser \\
\hline 3 & - & - & + & - & - & + & retrouver \\
\hline 4 & - & - & - & + & + & - & accaparer \\
\hline 5 & - & - & - & + & + & - & racheter \\
\hline 6 & - & - & - & + & + & - & contrôler \\
\hline 7 & - & - & - & + & + & - & annexer \\
\hline 8 & - & - & - & + & + & - & attirer \\
\hline 9 & - & - & - & - & + & + & confisquer \\
\hline 10 & - & - & - & - & + & + & arrêter \\
\hline 11 & - & - & - & - & - & + & consulter \\
\hline 12 & - & - & - & - & - & + & découvrir \\
\hline
\end{tabular}

On observe que la séquence polylexicale étudiée est en usage de façon libre, c'est-àdire lorsque les traits inhérents sont actualisés [1] et [2]. La séquence est figée lorsque les traits inhérents cessent d'être actualisés au profit de traits afférents; c'est le cas de toutes les autres occurrences répertoriées ci-dessus.

La séquence figée serait donc un dérivé tropologique, métaphorique ou métonymique, de l'expression libre. Apparemment, la séquence figée est produite à partir de 
la séquence libre dont elle conserve la motivation, même si celle-ci est entièrement virtualisée. La motivation reste transparente en [3], d'ailleurs un des traits inhérents - la préhension - se trouve actualisé. De fait, si on ne parvient plus à mettre la main sur ses clés, cela signifie implicitement qu'on ne peut physiquement poser la main, vue comme l'organe de préhension, sur les clés en question, parce qu'on ne sait plus où elles sont. C'est d'un mécanisme d'inférence qu'émerge l'idée qu'on les a égarées puisqu'on n'arrive plus à les retrouver. Toutefois, dans ce cas, on peut parler de motivation transparente sans pour autant admettre une actualisation évidente des autres traits inhérents: main en tant que prolongement du bras et organe du toucher. On peut se réjouir, par exemple, d'avoir fini par mettre la main sur ses clés alors que tout simplement on les a vues sans les toucher.

La motivation reste encore assez transparente en [4] où la représentation mentale induite par la séquence figée n'est pas sans rappeler le personnage de l'Avare de Molière qui serre contre lui sa cassette. Dans... mettre la main sur le magot, le mouvement du bras qui va permettre à la main de s'abattre sur un sac de pièces d'or et de le prendre est certes virtuel, mais sous-jacent à la construction du sens de l'énoncé: ... sa sœur n'attirait le vieux que dans le calcul de mettre la main sur le magot soupçonné. Toutefois, le magot dont il est question ici est en fait l'héritage d'un vieil homme, comme l'indique la présence dans l'énoncé de sœur, attirer le vieux, calcul, magot soupçonné. Or, le patrimoine dont on peut hériter ne se compose pas nécessairement d'un sac de pièces d'or; à l'heure actuelle, la même expression figée peut être employée avec le même objet alors que l'héritage peut être composé de valeurs mobilières dématérialisées et de biens immobiliers, par exemple. Le geste de préhension est alors tout à fait absent.

La motivation s'opacifie progressivement dans les emplois suivants jusqu'à devenir tout à fait opaque dans les occurrences [11] et [12], où la représentation mentale ne fait plus intervenir de geste de la main, même symboliquement.

\section{Construction du sens}

On soupçonne la présence d'une métaphore lorsqu'on décèle une anomalie sémantique (Durieux 1998). Par exemple, en [8] ... mettre la main sur l'élite des scientifiques: il y a dissonance entre l'action de poser l'organe du toucher et de préhension qu'est la main sur un objet, et le fait que cet objet soit une équipe de chercheurs.

Une fois l'anomalie repérée, la démarche de construction du sens consiste à mettre en évidence une relation de forme indicielle. On relève des indices dans le contexte verbal: notamment le point d'interrogation appliqué à nombre suffisant de chercheurs, recrutement de chercheurs étrangers. Ces indices confirment l'idée d'une incertitude sur les possibilités pour les pays asiatiques de disposer des chercheurs dont ils ont besoin. Ensuite intervient la mise en évidence d'une relation de type inférentiel: si les pays asiatiques ne produisent pas dans leurs universités les chercheurs dont ils ont besoin et si la Californie offre des conditions financières attrayantes aux chercheurs, alors les pays asiatiques vont avoir du mal à les attirer et vont se trouver en situation de pénurie. Or, ce dont on manque acquiert une valeur élevée. À ce stade, c'est la mise en évidence d'une relation analogique qui prend le relais. Les chercheurs de pointe étant très peu nombreux et donc une ressource rare et très convoitée sont considérés comme un trésor. Il y a bien transfert analogique entre 
l'élite des scientifiques, nécessairement restreinte, et un trésor. On parvient ainsi à la substitution par similitude de trésor à élite des scientifiques. L'énoncé contenant l'expression figée devient alors: Les pays d'Asie auront alors plus de mal à mettre la main sur le/un trésor. Dans le cas de ce nouvel énoncé, la motivation de l'expression figée acquiert une plus grande transparence, la représentation mentale suscitée évoquant aisément le geste de la main qui s'abat sur un objet convoité - un trésor - pour le prendre dans un but d'appropriation. En fait cette démarche conduit à remonter le courant de la dérivation tropologique et à rapprocher progressivement l'expression figée de l'expression libre originelle.

Certes, il est tentant de décomposer le processus de lecture d'une expression figée métaphorique en une séquence logique d'opérations cognitives:

(1) repérage d'une anomalie sémantique;

(2) mise en évidence d'une relation indicielle;

(3) mise en évidence d'une relation inférentielle;

(4) mise en évidence d'une relation analogique.

Toutefois, d'une part, la démarche (i) ne comporte pas toujours toutes ces étapes, (ii) ne se déroule pas toujours dans cet ordre, et (iii) peut inclure des relations imbriquées multiples. D’autre part, si cette décomposition peut présenter un intérêt sur le plan descriptif, sa valeur pragmatique est moins évidente. En effet, il n'est pas certain que le lecteur - et le traducteur est un lecteur particulier - procède couramment de la sorte. Plus précisément, il ne passe consciemment par ces étapes successives que s'il a du mal à construire le sens de l'énoncé comportant l'expression figée, soit parce qu'il rencontre celle-ci pour la première fois (n'oublions pas que le traducteur est normalement confronté à un texte en langue étrangère qu'il doit rédiger dans sa langue maternelle), soit parce qu'il est confronté à un emploi nouveau pour lui d'une expression qui lui est par ailleurs familière, mais dans d'autres contextes.

En revanche, s'il connaît et reconnaît la séquence figée, il en saisit la signification d'emblée et appréhende globalement le sens de l'énoncé qui la contient. Ainsi, en [7], Il réussit à mettre la main sur la Macédoine en 294: les traits afférents de conquête, possession, annexion se trouvent clairement actualisés par (i) la présence dans le contexte du mot empire à deux reprises et des séquences efforts pour conserver, se maintenir, et (ii) le co-occurrent réussit à qui confère une connotation positive évidente à mettre la main sur.

Dans le processus de construction du sens, la signification de la séquence figée est fonction du contexte en même temps qu'elle confère aux unités contextuelles une signification propre. Il y a toute une série d'interactions entre les unités lexicales et leur co-texte et leur contexte (Durieux, à paraître). Ainsi, en [6] ... Suez et Vivendi veulent mettre la main sur l'eau, la santé, l'agriculture, l'énergie: le contexte de stratégie d'acquisition par des groupes industriels et financiers confère à la séquence mettre la main sur la valeur de prendre le contrôle par le biais de prises de participation au capital d'entreprises, et c'est la signification ainsi construite qui donne aux objets eau, santé, agriculture, énergie une dimension métonymique. On remarque que la relation métonymique est ici au moins à double détente: chacun de ces quatre mots désigne en fait (i) un secteur d'activité, et (ii) des sociétés appartenant à ces secteurs d'activité. Concrètement, il s'agit bien de la part de Suez et de Vivendi d'acheter des actions de ces sociétés en nombre suffisant pour détenir une part de 
capital leur permettant d'exercer un contrôle sur les activités et décisions de ces sociétés et, en fin de compte, sur les secteurs d'activité tout entiers dont elles font partie.

\section{Stratégie de traduction}

Une fois parvenu au terme de la phase de compréhension, le traducteur aborde la phase de réexpression. La tentation est alors grande d'envisager une formulation la plus proche possible de l'originale. Dans le passage du français à l'anglais, par exemple, mettre la main sur (quelque chose ou quelqu'un) appelle assez naturellement to put a hand on (something or somebody). Or, cette forme serait fautive dans tous les cas pour traduire les douze occurrences de l'expression mettre la main sur composant notre corpus. En effet, le Dictionary of Contemporary English (Longman 2003) propose entre autres formes figées:

[a] Get your hands on sth - to succeed in getting something: She is only marrying him to get her hands on his money.

[b] Lay your hands on sth - to find or get something: I could read any book I could lay my hands on.

[c] Get your hands on sb - to catch someone you are angry with: Just wait till I get my hands on you!

Ce que confirme l'International Dictionary of Idioms (Cambridge 1998). On remarque que (i) le verbe support n'est pas to put, (ii) le substantif hand n'est pas déterminé par un article mais par un adjectif possessif, et (iii) le substantif hand est au pluriel.

Parmi les autres figements répertoriés, on en trouve un avec to put et hand au singulier en co-occurrence:

[d] put your hand in your pocket - to give money to charity: People are more inclined to put their hands in their pockets to help children.

Toutefois, d'une part, l'expression comporte encore l'adjectif possessif et, d'autre part, sa signification est tout autre. L'expression correspondante en français serait: mettre la main à la poche.

On trouve bien un figement avec le substantif hand au singulier précédé de l'article indéfini en co-occurrence avec le verbe to lay (cf. [b]):

[e] lay a hand on sb - to hurt someone: If you lay a hand on her, I'll report to the police.

Là encore, la signification est très différente et correspondrait plutôt à l'expression figée française: lever la main sur quelqu'un.

Cela confirme qu'il y a lieu de considérer la séquence figée comme un ensemble d'unités lexicales indissociables les unes des autres et de la traiter globalement comme une unité sémantique simple. C'est donc la signification résultant d'interactions avec le co-texte et le contexte tant verbal que cognitif qui est retenue pour l'exécution de la traduction. Bref, la séquence verbale polylexicale figée est traitée en traduction comme le verbe simple présentant la signification correspondante.

Toutefois, comme le fait justement remarquer Mejri (1997), l'emploi d'une séquence verbale polylexicale figée plutôt que d'un verbe simple n'est pas fortuit et témoigne d'une volonté stylistique, souvent de renforcement ou d'emphase.

Dans le paradigme de la théorie interprétative de la traduction, le sens est certes l'objet de l'opération traduisante, mais à cet égard le sens est considéré comme la 
résultante de toute une série de paramètres: matière linguistique, contexte cognitif, références intertextuelles, situation de communication, etc. Si la forme du texte original en tant que telle n'est pas l'objet de la traduction, il n'en reste pas moins que l'effet de la forme fait partie intégrante de la construction du sens. Ainsi, par exemple en [4], ... mettre la main sur le magot soupçonné ne saurait donner lieu à la même traduction que... obtenir le magot soupçonné. La séquence verbale polylexicale est beaucoup plus forte que le verbe simple. Même accaparer, proposé comme verbe simple correspondant dans notre tableau, est plus faible que mettre la main sur qui, dans cet emploi, reflète l'envie, la cupidité, un désir d'appropriation et presque une préméditation de vol. Le choix de la formulation en langue d'arrivée - qu'il s'agisse d'une séquence polylexicale ou d'un verbe simple - doit intégrer ce différentiel d'intensité.

En résumé, il y a adéquation entre la séquence polylexicale figée présente dans le texte de départ et sa traduction si cette dernière répond à la somme des trois exigences suivantes: dénotation + connotation + effet.

Ainsi, par exemple, l'expression anglaise [a] traduit de façon adéquate l'occurrence [4] de notre corpus; d'ailleurs le contexte est similaire: captation d'héritage ou de fortune.

De même, l'expression anglaise [c] qui s'applique à une personne, traduira de façon adéquate l'occurrence [10] de notre corpus parce que la connotation négative est bien présente dans le cas de l'arrestation d'un terroriste; en revanche, elle ne conviendrait pas à la traduction de [11] parce que, bien que l'objet soit là aussi une personne, il n'y a aucune hostilité dans la recherche d'un pédiatre pour soigner un enfant malade un jour de grève des médecins.

L'expression anglaise [b], par exemple, recouvre pratiquement les mêmes dénotation, connotation et effet que ce qui ressort de l'occurrence [3]; toutefois, l'exemple que donne le dictionnaire Longman appellerait en français une traduction telle que:

[f] je lisais tous les livres qui me tombaient sous la main, plutôt que

[g] je lisais tous les livres sur lesquels je pouvais mettre la main.

La dénotation et la connotation semblent identiques, mais l'effet entre [f] et [g] est inverse. En [f], la dominante est la facilité: il était facile de lire, l'obtention des livres était facile, ils étaient à portée de main. En revanche, en [g], la dominante est la difficulté: il était difficile de lire, l'obtention des livres était difficile, il fallait les chercher.

On remarque que l'expression figée en anglais [b] présente des similitudes syntaxiques avec l'expression figée en français. D'une part, elle se construit avec le modal can, ce qui correspond à la construction en français avec pouvoir/arriver à/parvenir à. D’autre part, elle prend spontanément la forme négative:

[h] I can't lay my hands on my keys.

Je ne peux pas mettre la main sur mes clés.

Je n'arrive pas à mettre la main sur mes clés.

\section{Conclusion}

Cette étude pragmatique tend à confirmer plusieurs caractéristiques des séquences polylexicales figées :

- La signification de l'ensemble des unités lexicales constitutives de la séquence est autonome et non égale à la somme des significations des unités lexicales prises isolément. 
- Une séquence polylexicale figée est aussi polysémique qu’une unité unilexicale.

- La construction du sens de la séquence figée est dépendante du contexte qui luimême prend sens en fonction de la signification de la séquence figée.

En conséquence, le traducteur est amené à soumettre les séquences figées au même traitement que les unités unilexicales: interactions entre la matière linguistique et le contexte cognitif pour préciser les significations, mise en œuvre d'un processus inférentiel pour construire le sens, choix des formulations en langue d'arrivée en fonction de l'impact à exercer sur le lecteur.

\section{RÉFÉRENCES}

Durieux, C. (1991): «La créativité en traduction technique», TextContext, Heidelberg, pp. 9-20.

Durieux, C. (1998): «Le Figement lexical: Approche cognitive de l'appréhension du sens», in Le Figement lexical, CERES, Tunis, pp. 133-143.

Durieux, C. (2004): «Complexité et cognition: un paradigme pour la traductologie», Equivalences 32-2, Bruxelles, pp. 5-30.

MejRi, S (1997): Le Figement lexical, Publications de la Faculté des lettres de la Manouba, Tunis.

MejRi, S. (1998): «Structuration sémantique et variation des séquences figées», Le figement lexical, CERES, Tunis, pp. 103-112.

Rastier, F. (1996): Sémantique interprétative, PUF, Paris. 